\title{
Novel VRK1 Mutations in a Patient with Childhood-onset Motor Neuron Disease
}

\author{
Genpei Yamaura ${ }^{1}$, Yuichi Higashiyama ${ }^{1}$, Kaori Kusama ${ }^{1}$, Misako Kunii ${ }^{1}$, Kenichi Tanaka ${ }^{1}$, \\ Shigeru Koyano ${ }^{1}$, Mitsuko Nakashima ${ }^{2,3}$, Yoshinori Tsurusaki ${ }^{2}$, Noriko Miyake ${ }^{2}$, \\ Hirotomo Saitsu ${ }^{2,3}$, Yukiko Iwahashi ${ }^{1}$, Hideto Joki ${ }^{1}$, Naomichi Matsumoto ${ }^{2}$, \\ Hiroshi Doi ${ }^{1}$ and Fumiaki Tanaka ${ }^{1}$
}

\begin{abstract}
:
A 24-year-old Japanese man exhibited slowly progressive gait disturbance from childhood to young adulthood. Physical and physiological examinations showed the involvement of both upper and lower motor neurons, fulfilling the diagnostic criteria for amyotrophic lateral sclerosis (ALS). Mild cognitive impairment and subclinical sensory involvement were also observed. A genetic analysis revealed novel compound heterozygous mutations, c.767C $>\mathrm{T}$ (p.Thr256Ile) and c.800A >G (p.Asp267Gly), in the vaccinia-related kinase 1 gene (VRK1). This is the first report of a Japanese patient with a motor neuron disease phenotype caused by VRKI mutations. This diagnosis should be considered in atypical cases of juvenile-onset and slowly progressive types of motor neuron disease.
\end{abstract}

Key words: motor neuron disease, amyotrophic lateral sclerosis, VRK1, childhood-onset

(Intern Med 58: 2715-2719, 2019)

(DOI: 10.2169/internalmedicine.2126-18)

\section{Introduction}

Motor neuron diseases (MNDs) are clinically and genetically heterogeneous disorders associated exclusively or predominantly with dysfunction of upper and/or lower motor neurons (1). Amyotrophic lateral sclerosis (ALS), a representative MND, is characterized by both upper and lower motor neuron involvement, and typically follows a rapidly progressive fatal clinical course (2). However, atypical forms of MNDs, including ALS, variably affect the upper and lower motor neurons and patients with these conditions exhibit extra-motor signs and/or slow progression (3).

Mutations in the vaccinia-related kinase 1 gene (VRK1) have been reported to cause an atypical, infantile-onset lower MND phenotype [i.e., spinal muscular atrophy (SMA)], which is associated with pontocerebellar hypoplasia as well as death in late childhood (4). Moreover, some pa- tients have demonstrated clinical upper motor neuron signs mimicking ALS $(5,6)$. We herein present a 24-year-old Japanese man with childhood-onset mild MND that fulfilled the diagnostic criteria for ALS, and who had novel compound heterozygous mutations in VRK1. This study describes the first Japanese case of MND with VRK1 mutations.

\section{Case Report}

The patient was a 24-year-old Japanese man who was born with a normal birthweight and whose motor developmental milestones were almost normal, including stable head control at 4 months and acquisition of walking at 14 months. He had no notable family history with the exception of a paternal cousin with intellectual disability and autism without motor involvement (Fig. 1a).

At around 3 years of age, his family suspected that he

\footnotetext{
${ }^{1}$ Department of Neurology and Stroke Medicine, Yokohama City University Graduate School of Medicine, Japan, ${ }^{2}$ Department of Human Genetics, Yokohama City University Graduate School of Medicine, Japan and ${ }^{3}$ Department of Biochemistry, Hamamatsu University School of Medicine, Japan

Received: September 13, 2018; Accepted: March 26, 2019; Advance Publication by J-STAGE: June 7, 2019

Correspondence to Dr. Fumiaki Tanaka, ftanaka@yokohama-cu.ac.jp
} 


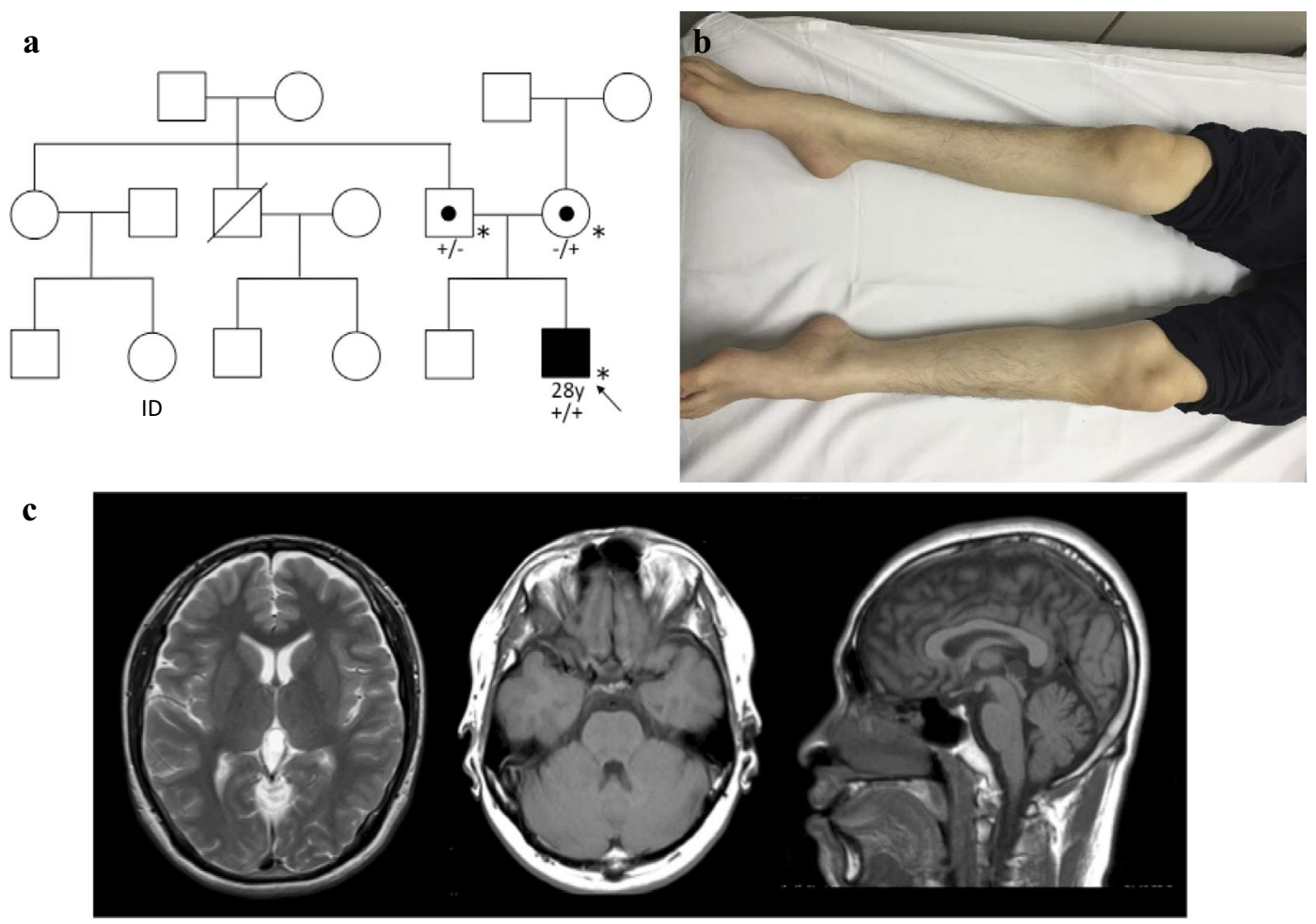

Figure 1. The clinical presentation of the patient. (a). The family pedigree. Individuals with available blood samples are indicated by an asterisk (*). The VRK1 compound heterozygous mutation is indicated by $+/+$. The heterozygous mutation is indicated by +/- or $-/+$. ID: intellectual disability (b). Photograph of the lower limbs. There was atrophy of the distal limbs, especially the lower legs. (c). Brain MRI findings. T2- (left) and T1-weighted imaging (middle and right). There were no abnormal findings, with the exception of a pineal cyst. There was no pontocerebellar hypoplasia.

had mild intellectual disability, which was confirmed by intelligence testing at 6 years of age. At 7 years of age, he developed difficulty in running, and gait instability was noted approximately 2 years later. He was admitted to a nearby hospital and spasticity was observed in his legs. However, the cause of the symptoms remained unknown. After graduation from a regular high school class at 18 years of age, he became aware of weakness in his lower legs, leading to difficulty in walking. At 23 years of age, he developed difficulty in climbing stairs and fell occasionally. He also noticed weakness in his grip strength in the same year.

Neuromuscular diseases such as cerebral palsy, ALS, and SMA were suspected at that time, and genetic testing for SMA, including the survival of motor neuron 1 (SMN1) and NLR family apoptosis inhibitory protein (NAIP) mutations, was conducted, but no mutations were found in these genes.

At 24 years of age, he was admitted to our hospital. On physical examination, he had prominent muscular atrophy that particularly affected his lower legs, including the tibialis anterior and gastrocnemius muscles (Fig. 1b). Manual muscle testing showed symmetrical weakness predominantly in his distal limbs; the Medical Research Council (MRC) scores of the proximal muscles of the upper and lower limbs were $5 / 5$ and $4 / 5$, respectively, while those of the distal muscles were $3 / 5$ and $1 / 5$, respectively. The patellar tendon reflexes were brisk, and tendon reflexes in the upper limbs were slightly increased. He showed steppage gait due to weakness of the tibialis anterior muscles. There were no fasciculations, pathological signs (e.g., extensor plantar reflexes), sensory symptoms, or cerebellar ataxia. His MiniMental State Examination score was 23/30, indicating mild cognitive impairment.

The patient's serum creatine kinase level was mildly elevated [488 U/L (normal range: 40-265 U/L)]. Needle electromyography (EMG) showed chronic neurogenic changes in the upper and lower limb muscles; the rectus femoris and extensor carpi radialis muscles showed muscle unit potentials (MUPs) with high amplitude and a long duration, as well as reduced recruitment of MUPs without spontaneous activity at rest. The mentalis and sternocleidomastoid muscles showed normal MUPs. In a nerve conduction study, compound muscle action potentials (CMAPs) exhibited low amplitudes in the median and peroneal nerves without any reduction in motor conduction velocities. The sensory nerve action potentials (SNAPs) of the ulnar and sural nerves showed low amplitudes (Table).

Muscle CT revealed mild atrophy in the thigh and severe atrophy in the lower legs. On brain MRI, a pineal cyst was incidentally detected, but cerebellar hypoplasia was not found (Fig. 1c).

The patient's respiratory function was normal (forced expiratory volume $1.0 \%=91.5 \%, \%$ vital capacity $=83.6 \%$ ), and 
electrocardiography and echocardiography revealed no evidence of cardiac involvement.

The patient's physiological findings suggested the involvement of both the upper and lower motor neurons in the cervical and lumbosacral segments of the spinal cord, fulfilling the revised El Escorial criteria for probable ALS (7). However, the slowly progressive course, mild intellectual disability beginning in childhood, and mild electrophysiological sensory nerve involvement were atypical for ALS.

To identify the causative genetic mutations, whole-exome sequencing was performed, as previously described (8). Genomic DNA was processed using a SureSelect Human All Exon Kit v5 (Agilent Technologies, Santa Clara, USA). Captured DNAs were sequenced using a HiSeq2500 (Illuma-

Table. Nerve Conduction Study Results.

\begin{tabular}{lccc}
\hline Motor nerve & & & \\
\hline & DL $(\mathrm{ms})$ & NCV $(\mathrm{m} / \mathrm{s})$ & CMAP $(\mathrm{mV})$ \\
R. Median & $4.0(<4.2)$ & $51(>48)$ & $1.2(>3.5)$ \\
R. Ulnar & $2.7(<3.4)$ & $64(>49)$ & $5.4(>2.8)$ \\
R. Tibial & $4.0(<6.0)$ & $50(>41)$ & $5.7(>2.9)$ \\
R. Peroneal & N.E. $(<5.5)$ & N.E. $(>40)$ & N.E. $(>2.5)$ \\
\hline Sensory nerve & & \\
\hline \multicolumn{5}{l}{ DL $(\mathrm{ms})$} & NCV $(\mathrm{m} / \mathrm{s})$ & SNAP $(\mu \mathrm{V})$ \\
R. Median & $2.2(<3.5)$ & $65(>47)$ & $26(>20)$ \\
R. Ulnar & $2.0(<3.1)$ & $65(>44)$ & $10(>18)$ \\
R. Sural & $3.4(<3.4)$ & $43(>41)$ & $2(>10)$ \\
\hline
\end{tabular}

Median nerve stimulation shows low CMAP in the adductor pollicis brevis muscle, and low SNAP in the sural nerve. Normal limits are indicated in parentheses. DL: distal latency, NCV: nerve conduction velocity, CMAP: compound muscle action potential, N.E.: not evoked nia, San Diego, USA). We checked whether the genes related to spastic paraplegia, ALS/frontotemporal lobar degeneration, hereditary motor neuronopathies (HMN), or SMA exhibited any rare variants (minor allele frequency $<1 \%$ in our database of 575 normal Japanese exomes). Among these, we identified potentially pathogenic homozygous or compound heterozygous variants for autosomal recessive diseases and heterozygous variants for dominant diseases.

Only two variants of VRK1 were detected as potential causative mutations for the disease. Sanger sequencing of the patient and his parents confirmed the existence of VRK1 mutations in the patient (NM_003384) c.767C $>$ T (p.Thr256Ile) and c.800A $>$ G (p.Asp267Gly), and each parent had one of the mutations, suggesting autosomal recessive inheritance (Fig. 2a). Neither mutation was registered in the Exome Aggregation Consortium (ExAC) database (http:// exac.broadinstitute.org/) or in our in-house database of 575 normal Japanese exomes. The mutations were predicted to be possibly damaging (score $=0.726$, sensitivity: 0.86 , specificity: 0.92) and probably damaging (score $=0.998$, sensitivity: 0.27, specificity: 0.99) by PolyPhen-2 (http://genetics.bw h.harvard.edu/pph2/), respectively, and to be disease-causing by Mutation Taster (www.mutationtaster.org/), but neutral $($ score $=-0.511)$ and deleterious $($ score $=-5.046)$ by PROVEAN (http://provean.jcvi.org/index.php), respectively. The conservation and location of the mutated amino acids are shown in Fig. 2b and c, respectively. Amino acid Asp 267 was evolutionally highly conserved, while the degree of conservation was somewhat lower at amino acid Thr256 (Fig. 2b). The distribution of reported and currently identified VRK1 mutations did not show a specific "hotspot" (Fig. 2c).

Although the mutations had not been reported previously,

b

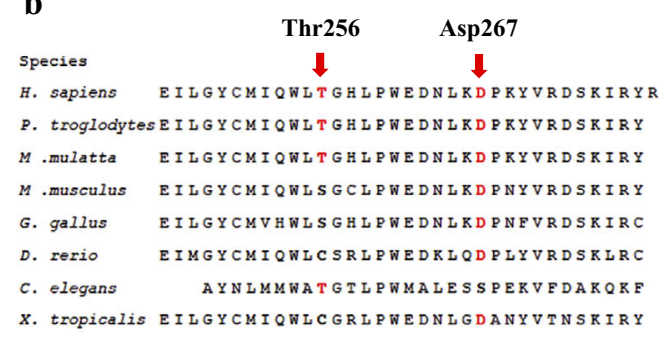

x. tropicalis EILGYCMIQWLCGRLPWEDNLGDANYVTNSRIRY
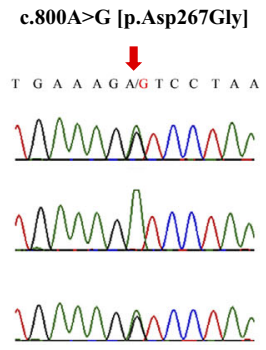

Mother

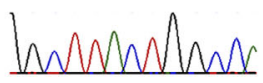
G G C T T A CT T G G C C monnlawe Inomolum hind
c.767C $>$ T [p.Thr256Ile]

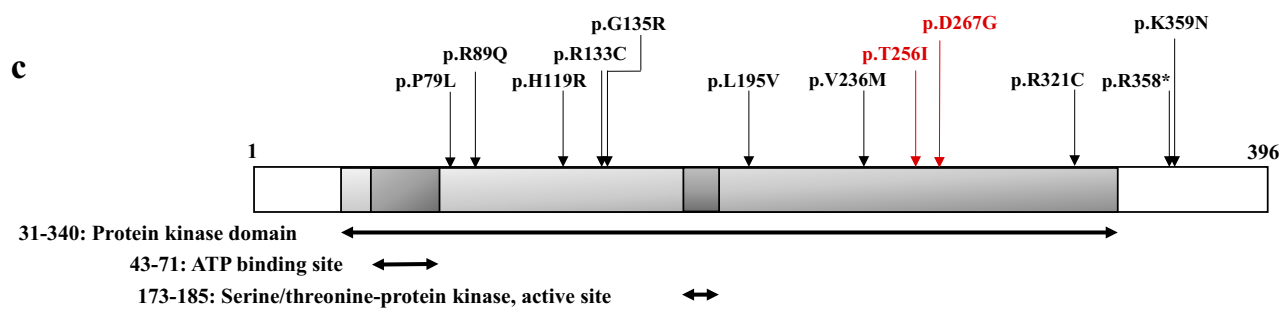

Figure 2. The VRK1 mutations of the patient. (a) An electropherogram of the genomic VRK1 sequence. Two mutations were detected in VRK1. Arrows indicate VRK1 c.767C $>T$ (p.Thr256lle) and c.800A > G (p.Asp267Gly) mutations. (b) Conservation of amino acids Thr256 and Asp267. (c) A schematic illustration of the structure and mutations of the VRK1 protein. Black and red arrows indicate the locations of known mutations and the mutations identified in this case, respectively. 
we considered them to be causative based on the results of amino acid-level prediction algorithms, the conservation of amino acids, and the fact that the patient's phenotype was consistent with previous cases of VRK1 mutations.

\section{Discussion}

We reported the case of a 24-year-old Japanese man who presented with a mild ALS-like phenotype of MND during childhood with novel compound heterozygous mutations in $V R K 1$. This is the first Japanese case of an MND phenotype caused by VRK1 mutations. VRK1 was identified as a causative gene for infantile-onset SMA with pontocerebellar hypoplasia in a consanguineous family of Ashkenazi Jewish origin (c.1072C >T; p.Arg358*) (4). However, recent studies have shown that mutations in this gene could cause a broader spectrum of MND, including ALS and motor neuropathy (6). VRK1 mutations were reported by Nguyen et al. in a case of slowly progressive adult-onset ALS (c.356A>G; p.His119Arg and c.961C $>$ T; p.ArgR321Cys) (5), and Stoll et al. reported two families: one with childhood-onset ALS with short stature and microcephaly (c.403G >A; p.Gly135 Arg and c.583T>G; p.Leu195Val); the other with childhoodonset SMA with mild to moderate generalized brain atrophy (c.356A >G; p.His119Arg and c.1072C >T; p.Arg358*) (6). VRK1 mutations do not necessarily cause MND, and patients with only moderate to severe intellectual disability and pontocerebellar hypoplasia (c.397C >T; p.Arg133Cys) have been reported (9). Our patient presented with upper motor neuron signs and physiological findings corresponding to lower motor neuron degeneration, which met the diagnostic criteria for ALS; however, he also showed extra-motor signs such as intellectual disability and subclinical sensory nerve involvement, which was evidenced by low-amplitude SNAPs. Although ALS and SMA (excluding type I SMA) do not affect sensory nerves (10), a patient with complex motor and sensory axonal neuropathy with VRK1 mutations (c.706G>A, p.V236M; and c.266G>A, p.R89Q) was described (11). Mutations of VRK1 can affect both the motor and sensory systems, but it remains unclear whether the reduction of SNAPs observed in our patient was due to axonal neuropathy or neuronopathy.

VRK1 is a ubiquitously expressed serine-threonine kinase that is known to play multiple roles, for instance in cellular proliferation, cell cycle regulation, histone modification, DNA repair, disruption of RNA processing, and carcinogenesis (12). As a kinase, VRK1 phosphorylates several proteins such as p53, barrier-to-autointegration factor 1 (BANF1), histone H3, and CREB, all of which are involved in cell cycle regulation (12). A recent report on partial VRK 1 knockout mice clarified the associated motor phenotype and identified significant changes of expression in the gene sets that predict novel roles for VRK1, such as neurotrophin signaling, axon guidance, proteasome-related genes, and oxidative phosphorylation, all of which have been implicated in the pathogenesis of ALS (13). However, the specific path- way of VRK1 dysfunction that contributes to motor neuron degeneration remains to be elucidated.

In conclusion, we described the case of a Japanese patient harboring novel VRK1 mutations associated with mild, and slowly progressive form of ALS-like MND with subclinical sensory involvement, the onset of which occurred in childhood. Our patient demonstrated the broad phenotypic spectrum of VRK1 mutations, and further studies should be performed to investigate the role of this gene in the differential diagnosis of atypical MNDs with juvenile onset and slow progression.

\section{The authors state that they have no Conflict of Interest (COI).}

Hiroshi Doi and Fumiaki Tanaka contributed equally to this work.

\section{References}

1. Finsterer J, Burgunder JM. Recent progress in the genetics of motor neuron disease. Eur J Med Genet 57: 103-112, 2014.

2. Statland JM, Barohn RJ, McVey AL, Katz J, Dimachkie MM. Patterns of weakness, classification of motor neuron disease and clinical diagnosis of sporadic ALS. Neurol Clin 33: 735-748, 2015.

3. De Jonghe P, Auer-Grumbach M, Irobi J, et al. Autosomal dominant juvenile amyotrophic lateral sclerosis and distal hereditary motor neuronopathy with pyramidal tract signs: synonyms for the same disorder? Brain 125: 1320-1325, 2002.

4. Renbaum P, Kellerman E, Jaron R, et al. Spinal muscular atrophy with pontocerebellar hypoplasia is caused by a mutation in the VRK1 gene. Am J Hum Genet 85: 281-289, 2009.

5. Nguyen TP, Biliciler S, Wiszniewski W, Sheikh K. Expanding phenotype of VRK1 mutations in motor neuron disease. J Clin Neuromuscul Dis 17: 69-71, 2015.

6. Stoll M, Teoh H, Lee J, et al. Novel motor phenotypes in patients with VRK1 mutations without pontocerebellar hypoplasia. Neurology 87: 65-70, 2016.

7. Brooks BR, Miller RG, Swash M, Munsat TL. World federation of neurology research group on motor neuron diseases. El Escorial revisited: revised criteria for the diagnosis of amyotrophic lateral sclerosis. Amyotroph Lateral Scler Other Motor Neuron Disord 1: 293-299, 2000.

8. Iwama K, Osaka H, Ikeda T, et al. A novel SLC9A1 mutation causes cerebellar ataxia. J Hum Genet 63: 1049-1054, 2018.

9. Najmabadi $\mathrm{H}, \mathrm{Hu} \mathrm{H}$, Garshasbi $\mathrm{M}$, et al. Deep sequencing reveals 50 novel genes for recessive cognitive disorders. Nature 478: 5763, 2011.

10. Rudnik-Schoneborn S, Goebel HH, Schlote W, et al. Classical infantile spinal muscular atrophy with SMN deficiency causes sensory neuronopathy. Neurology 60: 983-987, 2003.

11. Gonzaga-Jauregui C, Lotze T, Jamal L, et al. Mutations in VRK1 associated with complex motor and sensory axonal neuropathy plus microcephaly. JAMA Neurol 70: 1491-1498, 2013.

12. Valbuena A, Sanz-Garcia M, Lopez-Sanchez I, Vega FM, Lazo PA. Roles of VRK1 as a new player in the control of biological processes required for cell division. Cell Signal 23: 1267-1272, 2011.

13. Vinograd-Byk H, Renbaum P, Levy-Lahad E. Vrkl partial knockdown in mice results in reduced brain weight and mild motor dysfunction, and indicates neuronal VRK1 target pathways. Sci Rep 8: 11265, 2018. 
The Internal Medicine is an Open Access journal distributed under the Creative

by-nc-nd/4.0/). Commons Attribution-NonCommercial-NoDerivatives 4.0 International License. To view the details of this license, please visit (https://creativecommons.org/licenses/

(C) 2019 The Japanese Society of Internal Medicine Intern Med 58: 2715-2719, 2019 